\title{
Synthesis and Characterizations of Graft Copolymer of Ethylcellulose with Poly(caprolactone monoacrylate)
}

\author{
By Dongshan WANG, ${ }^{1,2}$ Changzhong CHEN, ${ }^{1,3}$ Yingnan XUAN, ${ }^{1}$
} Yong HUANG, ${ }^{1,4, *}$ and Jiarui SHEN ${ }^{2}$

Ethylcellulose grafting polycaprolactone monoacrylate copolymer (EC-g-PCLA) was synthesized by a two-step reaction of ethylcellulose (EC) with poly(caprolactone monoacrylate) (PCLA). The isocyanate-terminated intermediate (NCOPCLA) was prepared firstly and then it was grafted onto ethylcellulose chains. The structure analysis of the copolymers from the FTIR, ${ }^{1} \mathrm{H}$ NMR and ${ }^{13} \mathrm{C}$ NMR indicated that PCLA was connected to EC by chemical bonding. The flow temperature of graft copolymer was lower than that of the pure EC and decreased with increasing the grafting percentage. Outdoor soil burial and active sludge tests indicated that the graft copolymer has biodegradability in natural conditions.

KEY WORDS: Ethylcellulose / Graft / Polymer Synthesis / Characterization / Degradable /

Much attention has been given to the environmental pollution from non-degradable plastic wastes. Cellulose material $^{1}$ is an attractive and economically feasible alternative material in developing degradable plastics. ${ }^{2}$ A cellulose graft copolymer is an example of modification of the cellulose molecule ${ }^{3}$ by chemical derivation for use in thermoplastic production. $^{4-6}$ Ethylcellulose $^{7}$ (EC) is one of the most important cellulose ethers. It was used widely in various fields such as textile, membrane separation, and paint etc. However, this material has a high flow temperature $\left(T_{\mathrm{f}}\right)$, which results in the poor processing ability compared to typical plastics. It is believed that introduction flexible segments by chemical bonds on EC backbone may improve the processing ability. AbedelRazik $^{8}$ reported copolymerization of ethyl acrylate, methyl acrylate onto EC. Shen ${ }^{9}$ grafted EC with PS and PMMA. Aggour $^{10,11}$ reported radical graft reaction of EC. In this article, a novel graft copolymer was prepared by introducing flexible poly(caprolactone monoacrylate) (PCLA) chains onto EC backbone. The thermal and mechanical properties and biodegradability of EC-g-PCLA were discussed, respectively.

\section{EXPERIMENTAL}

\section{Materials}

Ethylcellulose $\left(\mathrm{EC}, M_{\mathrm{n}}=3.3 \times 10^{4}\right.$, DS $=2.1$ ) was supplied by Luzhou Chemical Company. Tolune-2,4 diisocyanate (TDI) was supplied by Tokyo Chemical Industry Company. Dibutyltin dilaurate (DBTDL) was supplied by Fluka Company. Polycaprolactone monoacrylate (PCLA) was supplied by Union Carbide Company and its molecular mass was 344. Other chemical reagents were analytically pure.

\section{Preparation of End Isocyanate Intermediate NCOPCLA}

TDI $(0.01 \mathrm{~mol})$ and acetone $(15 \mathrm{~mL})$ were placed into a three-neck flask and mixed by stirring. Then, $0.01 \mathrm{~mol}$ of PCLA and $15 \mathrm{~mL}$ acetone were added at $60^{\circ} \mathrm{C}$. The reaction began when $0.01 \mathrm{~mL}$ catalyst dibutyltin dilaurate (DBTDL) was added. The reaction was maintained for $3 \mathrm{~h}$. The reaction product was coagulated in hexane, washed with pentane and then filtered. The white powder intermediate NCOPCLA was obtained in almost quantitative yield (Scheme 1 (I)).

\section{Preparation of EC- $g$-PCLA}

Intermediate NCOPCLA was added to a three-neck flask containing quantitative EC and acetone at $60^{\circ} \mathrm{C}$. Then $0.01 \mathrm{~mL}$ catalyst DBTDL was added in the flask and the reaction was maintained for $10 \mathrm{~h}$. The reaction product was coagulated in ethanol and filtered. Then, it was extracted in chloroform for $24 \mathrm{~h}$ to remove the residual raw materials and NCOPCLA. The product was dissolved in acetone and cast on a glass gasket, which settled for $12 \mathrm{~h}$. The dried pure graft copolymer film was obtained after further dried under vacuum for $24 \mathrm{~h}$ (Scheme 1 (II)). The grafting percentage $(\% G)$ and weight percent of PCLA in graft copolymers $\left(\% W_{\text {PCLA }}\right)$ were calculated by the following equations:

$$
\begin{aligned}
& \% G=\left(W_{1}-W_{0}\right) \times 100 / W_{0} \\
& \% W_{\text {PCLA }}=\left(W_{1}-W_{0}-W_{2}\right) \times 100 / W_{1}
\end{aligned}
$$

where $W_{0}, W_{1}$ and $W_{2}$ are the weight of EC, graft polymer and TDI, respectively.

\section{Characterization}

The Fourier transform infrared (FT-IR) spectra of the raw materials and the graft copolymers were measured with a

\footnotetext{
${ }^{1}$ Key Laboratory of Cellulose and Lignocellulosics Chemistry, Guangzhou Institute of Chemistry, Chinese Academy of Sciences, Guangzhou 510650, China

${ }^{2}$ College of Material Science and Engineering, South China University of Technology, Guangzhou 510641, China

${ }^{3}$ Graduate School of Chinese Academy of Sciences, Beijing, 100049, China

${ }^{4}$ State Key Laboratory of Polymer Physics and Chemistry, Institute of Chemistry, Chinese Academy of Sciences, Beijing 100080, China

*To whom correspondence should be addressed (Tel: +86-10-82543478, E-mail: yhuang@ cashq.ac.cn).
} 


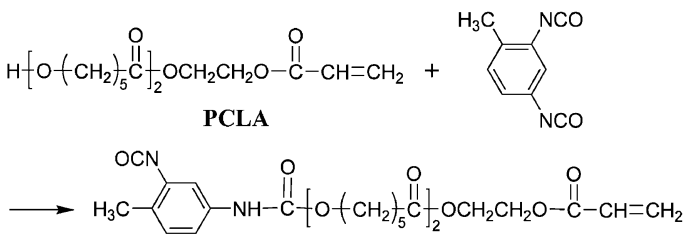

(I)

NCOPCLA

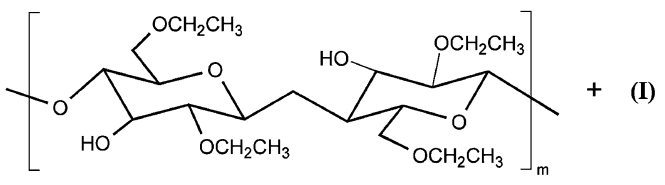

EC

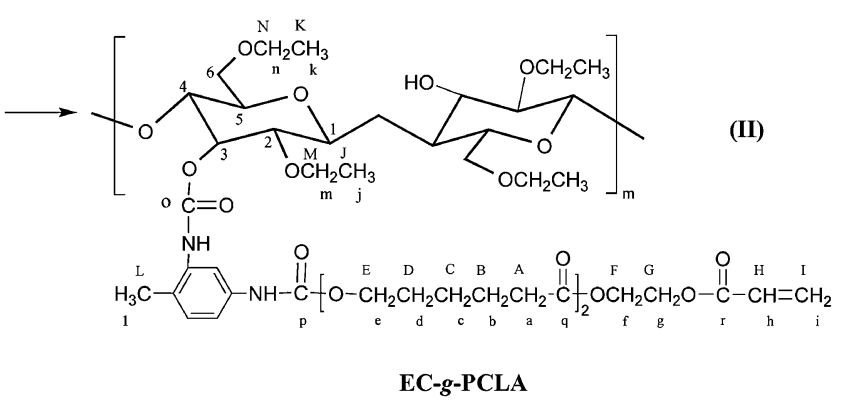

Scheme 1. Reaction scheme of EC-g-PCLA.

spectrophotometer (RFX-65, Analect Company, USA), using $\mathrm{KBr}$ pellets in the range of $400-4000 \mathrm{~cm}^{-1}$. ${ }^{1} \mathrm{H} \mathrm{NMR}$ and ${ }^{13} \mathrm{C}$ NMR spectra were obtained at $25^{\circ} \mathrm{C}$ with a spectrometer (DRX 400, Bruker, USA), using $\mathrm{CO}\left(\mathrm{CD}_{3}\right)_{2}$ as the solvent and trimethylsilane (TMS) as the internal standards.

The molecular mass of the graft copolymers were determined by gel permeation chromatography (GPC, Waters 551). Tetrahydrofuran (THF) was used as the mobile phase at a flow rate of $1 \mathrm{~mL} / \mathrm{min}$ and polystyrene (PS) standards were used to obtain the universal calibration curve.

The mechanical properties of the graft copolymers were measured by a universal testing machine (AG-I, Shimadzu Company, Japan), according to ASTM D882 with tensile speed of $50 \mathrm{~mm} / \mathrm{min}$.

The apparent flow temperature of EC and the copolymer were measured by a hot stage (STC200, Instec, USA). The sample was put on a glass sheet in the hot stage. Firstly the sample was heated at a $10^{\circ} \mathrm{C} / \mathrm{min}$ rate to obtain the approximate melting point, and then it was heated to the temperature $10^{\circ} \mathrm{C}$ below the melting point quickly, then the heating rate decreased to $1^{\circ} \mathrm{C} / \mathrm{min}$. When the solid sample is melt to liquid and begin flow, the flow temperature could be obtained from the data display panel of the hot stage.

The EC and EC-g-PCLA graft copolymers films were put into the soil and active sludge for degradation for different periods. Weight loss rate of the samples during the degradation in the soil burial and the active sludge was calculated according to the following formula, ${ }^{12}$

$$
\text { Weight loss rate }(\%)=\left(G_{0}-G_{\mathrm{i}}\right) / G_{0} \times 100
$$

Table I. The relationship of graft copolymers and reaction conditions

\begin{tabular}{cccccccc}
\hline & $\begin{array}{c}\text { PCLA } \\
(\mathrm{mol})\end{array}$ & $\begin{array}{c}\text { TDI } \\
(\mathrm{mol})\end{array}$ & $\begin{array}{c}\text { EC } \\
(\mathrm{mol})\end{array}$ & $\begin{array}{c}\text { Cat } \\
(\mathrm{mL})\end{array}$ & $\begin{array}{c}\text { EC- } g \text {-PCLA } \\
(\mathrm{g})\end{array}$ & $\begin{array}{c}\mathrm{G} \\
(\%)\end{array}$ & $\begin{array}{c}\mathrm{W}_{\text {PCLA }} \\
(\%)\end{array}$ \\
\hline $\mathrm{G}-1$ & 0.01 & 0.01 & 0.000373 & 0.02 & 16.40 & 33.33 & 14.39 \\
$\mathrm{G}-2$ & 0.01 & 0.01 & 0.000525 & 0.02 & 21.80 & 26.60 & 13.03 \\
$\mathrm{G}-3$ & 0.01 & 0.01 & 0.000671 & 0.02 & 26.20 & 18.34 & 8.85 \\
\hline
\end{tabular}

where $G_{0}$ is weight of the sample before treatment, and $G_{\mathrm{i}}$ is weight of the sample after treatment for $i \mathrm{~d}$. Intrinsic viscosity $[\eta]$ of graft copolymers were measured by using an Ubbelohde capillary viscometer in the THF solutions. The temperature was controlled in a range of $30+0.02{ }^{\circ} \mathrm{C}$ during the measurement.

Notes are to be concerned with subjects of limited scope which require no further information in a future publication. Short Communications deal with significant findings worthy of urgent publication.

\section{RESULTS AND DISCUSSION}

PCLA has a hydroxyl group in the one end of the chain and an unsaturated group in the other end of the chains. OH groups on PCLA chains could be reacted with TDI and then, connect to EC chains. Unsaturated groups in the another end of the PCLA chains could not be reacted with NCO group of TDI. Only one end of PCLA chains, therefore, could be grafted on EC chains and EC-g-PCLA graft copolymers without any crosslinking between EC chains may be prepared.

\section{Synthesis}

In general, isocyanates can easily react with compounds containing active protons such as alcohol, amine and acid. ${ }^{13-15}$ Experimental results show that the reaction of TDI with PCLA is a slow reaction without catalyst, but it is the fast and exothermic reaction in the presence of DBTDL and gives a higher yield. The variations of the grafting percentage $G$ and the weight percent of PCLA in graft copolymer $\mathrm{W}_{\text {PCLA }}$ with the different EC usage are shown in Table I. Obviously, decrease of the EC usage is accompanied by an increase in the grafting percentage and an increase in the weight percent of PCLA in graft copolymers. It is interpreted that there is higher concentration of NCOPCLA for each OH group of EC when the EC usage is lower in the reaction system, which could be in favor of the grafting process.

\section{Structure Characteristics}

Figure 1 shows the FT-IR spectra of PCLA, EC and the graft copolymer EC-g-PCLA. In Figure 1a, PCLA shows a strong hydroxyl peak at $3500 \mathrm{~cm}^{-1}$ and a sharp peak at $1732 \mathrm{~cm}^{-1}$ for the internal ester groups, and EC shows a typical hydroxyl peak at approximately $3480 \mathrm{~cm}^{-1}$ from Figure $1 \mathrm{~b}$. The FT-IR spectrum of EC- $g$-PCLA in Figure 1c shows new peaks at $1734 \mathrm{~cm}^{-1}$, which indicates the internal ester linkage, at $1537 \mathrm{~cm}^{-1}$ for the amide $\mathrm{NH}$ of carbamate group, and at $1603 \mathrm{~cm}^{-1}$ for the aromatic ring from TDI. However, the peak 


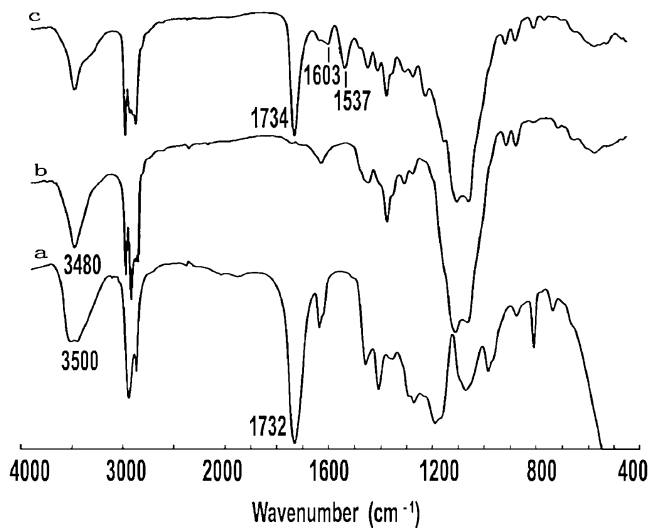

Figure 1. FT-IR spectra of (a) PCLA, (b) EC and (c) EC-g-PCLA (G-1).

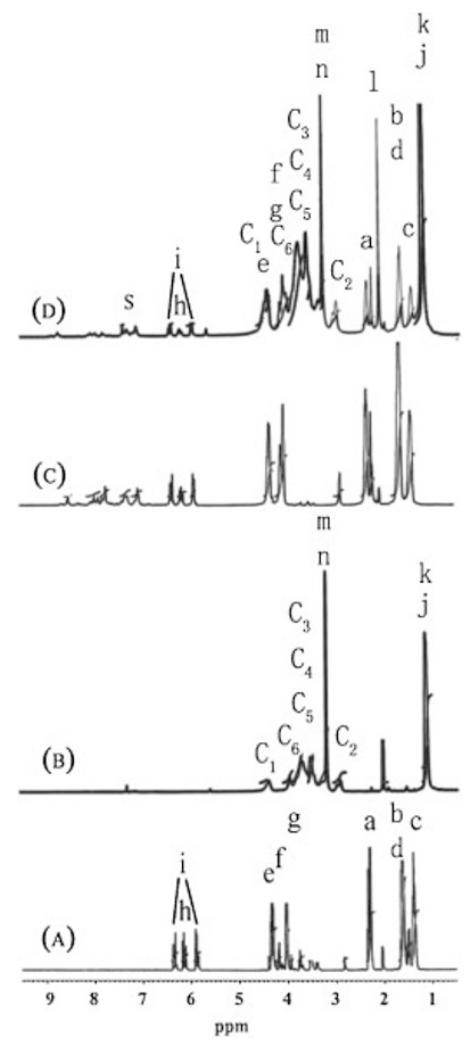

Figure 2. ${ }^{1} \mathrm{H}$ NMR spectra of (A) PCLA, (B) EC, (C) NCOPCLA and (D) EC$g$-PCLA (G-1).

intensity of the hydroxyl groups in EC- $g$-PCLA is reduced by the substitution of NCOPCLA compared with pure EC, which indicates that carbamate groups link EC and PCLA successfully.

Meanwhile, the ${ }^{1} \mathrm{H}$ NMR spectra of EC, PCLA, NCOPCLA and EC-g-PCLA are shown in Figure 2. From Figure 2A, PCLA shows hydrocarbon around 1.38-2.32 and 4.01$4.35 \mathrm{ppm}$, methylene around 5.89-5.91 and 6.34-6.38 ppm and hypo-methylene around $6.12-6.19 \mathrm{ppm}$. The proton spectrum of EC-g-PCLA (Figure 2D) shows the peaks of $\mathrm{EC}^{16}$ (Figure 2B) and shows methylene and hypo-methylene of PCLA. ${ }^{17}$ It also shows methyl of TDI resonance at $2.19 \mathrm{ppm}$,

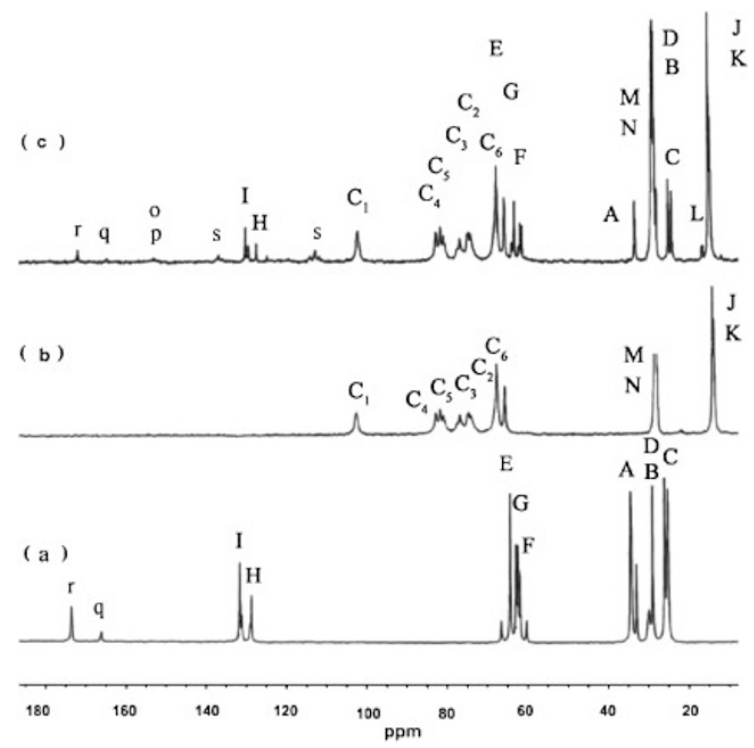

Figure 3. ${ }^{13} \mathrm{C}$ NMR spectrum of (a) PCLA, (b) EC and (c) EC-g-PCLA (G-1).

Table II. Molecular mass and $M_{w} / M_{n}$ of EC- $g$-PCLA copolymers

\begin{tabular}{ccccc}
\hline & $G(\%)$ & $M_{\mathrm{n}}(\mathrm{g} / \mathrm{mol})$ & $M_{\mathrm{w}}(\mathrm{g} / \mathrm{mol})$ & $M_{\mathrm{w}} / M_{\mathrm{n}}$ \\
\hline G-1 & 33.33 & 43,000 & 88,000 & 2.05 \\
G-2 & 26.60 & 36,000 & 80,000 & 2.18 \\
G-3 & 18.34 & 35,000 & 71,000 & 2.06 \\
EC & 0 & 33,000 & 85,000 & 2.54 \\
\hline
\end{tabular}

and benzene ring resonance at 7.04-7.47 ppm from NCOPCLA in Figure 2C. Apparently, the ${ }^{1} \mathrm{H}$ NMR spectra confirm the grafting PCLA onto EC.

Moreover, the carbon spectra ${ }^{18}$ of EC, PCLA and EC- $g$ PCLA is given in Figure 3. Compared with the ${ }^{13} \mathrm{C}$ NMR spectra of EC (Figure 3b) and PLCA (Figure 3a), the spectrum of the graft copolymer (Figure $3 \mathrm{c}$ ) shows not only the peaks of EC, but also several peaks appeared around 20.6-34.4 ppm representing the presence of the hydrocarbon chains of PCLA, at $173.6 \mathrm{ppm}$ from ester linkage and at $154.6 \mathrm{ppm}$ from carbonyl group of carbamate groups. It also shows aromatic groups with peaks at $17.3 \mathrm{ppm}$ from methyl group, and at 120 $140 \mathrm{ppm}$ from NCOPCLA. Therefore, the results of ${ }^{13} \mathrm{C}$ NMR spectra also suggest the grafting of PCLA onto EC backbone.

Table II shows the number-average molecular mass $M_{\mathrm{n}}$ and weight-average molecular mass $M_{\mathrm{w}}$ of EC and the EC- $g$-PCLA with different grafting percentage $\mathrm{G}$. It is clear that both $M_{\mathrm{n}}$ and $M_{\mathrm{w}}$ of the graft copolymer are increased with increasing the graft percentage $G$. The molecular mass distribution $M_{\mathrm{w}} / M_{\mathrm{n}}$, however, decreases with increasing graft percentage, which demonstrates that the molecular mass distribution of the copolymer becomes narrower than the pure EC.

\section{Processing Ability}

The flow temperature of polymers is a very important factor for its processing ability, and higher flow temperature results in the poor processing ability. In this work, the flow temperatures 
Table III. Mechanical and thermal properties of EC- $g$-PCLA copolymers

\begin{tabular}{ccccc}
\hline G (\%) & $\begin{array}{c}\text { Tensile Strength } \\
(\mathrm{MPa})\end{array}$ & $\begin{array}{c}\text { Elongation } \\
(\%)\end{array}$ & $\begin{array}{c}\text { Flow Temperature } \\
\left({ }^{\circ} \mathrm{C}\right)\end{array}$ \\
\hline G-1 & 33.33 & 30.19 & 25.45 & $138.0-140.0$ \\
G-2 & 26.60 & 33.45 & 24.24 & $142.0-144.0$ \\
G-3 & 18.34 & 37.68 & 21.82 & $148.0-150.0$ \\
EC & 0 & 38.43 & 9.09 & $168.0-170.0$ \\
\hline
\end{tabular}

Table IV. Properties of PCL, EC- $g$-PCL and EC- $g$-PCLA copolymers

\begin{tabular}{lcccrc}
\hline & $\begin{array}{c}T_{\max }{ }^{a} \\
\left({ }^{\circ} \mathrm{C}\right)\end{array}$ & $\begin{array}{c}\text { Tensile Strength } \\
(\mathrm{MPa})\end{array}$ & $\begin{array}{c}\text { Elongation } \\
(\%)\end{array}$ & $\begin{array}{c}M_{\mathrm{n}}{ }^{\mathrm{b}} \\
(\mathrm{g} / \mathrm{mol})\end{array}$ & $M_{\mathrm{w}} / M_{\mathrm{n}}{ }^{\mathrm{b}}$ \\
\hline $\mathrm{PCL}$ & 372.8 & 23.00 & 700 & 11200 & 1.28 \\
EC-g-PCL & 336.3 & $/$ & $/$ & 183200 & 1.52 \\
EC-g-PCLA(G-1) & 372.0 & 30.19 & 25.45 & 43000 & 2.05 \\
\hline
\end{tabular}

${ }^{a} T_{\max }$ is the temperature corresponding to the maximum rate of weight loss. ${ }^{\mathrm{b}} M_{\mathrm{n}}$ and $M_{\mathrm{w}} / M_{\mathrm{n}}$ are determined by GPC analysis with polystyrene standards.

of EC and the graft copolymer EC- $g$-PCLA are measured by a hot stage shown in Table III. It is clear to see that all the flow temperatures of three copolymers are lower than that of EC, and the flow temperature is decreased with increasing the grafting yield. This means that the interactions between the EC molecular chains are weakened and the rigidity of EC is reduced after PCLA is grafted onto the EC backbone. Therefore, the graft copolymers are more easily fluent than the pure EC, which would result in the improvement of processing ability of graft copolymers.

Table IV shows the temperature corresponding to the maximum rate of weight loss $T_{\max }$ of PCL, EC- $g$-PCL ${ }^{19}$ and EC- $g$-PCLA. It can be found from Table IV that the temperature corresponding to the maximum rate of weight loss $T_{\max }$ of the EC- $g$-PCLA copolymers is almost the same as that of PCL homopolymers, but that of EC-g-PCL copolymers is lower than that of both PCL and EC- $g$-PCLA. It is suggested that the thermal stability of EC- $g$-PCLA copolymers is higher than that of EC-g-PCL copolymers.

\section{Mechanical Properties}

Mechanical properties such as the tensile strength and the elongation of the copolymers are very important for polymer materials. Table III also shows the mechanical properties of EC and EC- $g$-PCLA copolymers. From the data in Table III, it can be found that the tensile strength of the grafting copolymers is lower than that of the pure EC and is decreased with increasing the graft percentage $\mathrm{G}$. This suggests again that the interactions between the EC molecular chains are weakened after the grafting of PCLA onto the EC backbone, and intermolecular hydrogen bonding is destroyed. However, the elongation of the graft copolymers is larger than that of the pure EC and is increased with increasing the graft percentage $\mathrm{G}$, which indicates the tenacity of the EC material is improved after the PCLA flexible segments are introduced to EC backbone. It can be found, from Table IV, that the tensile strength of EC-g-PCLA copolymers is also higher than that of a

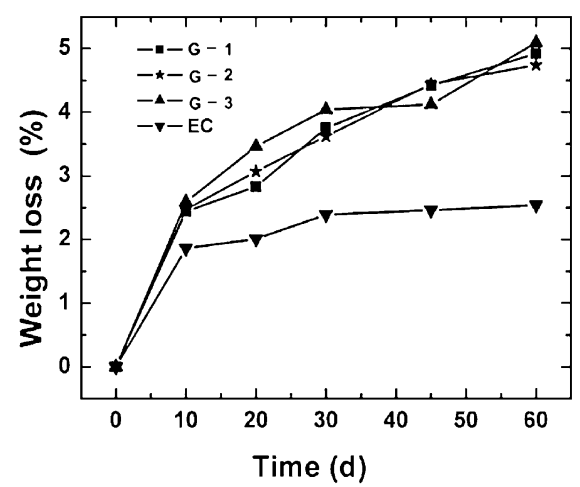

b

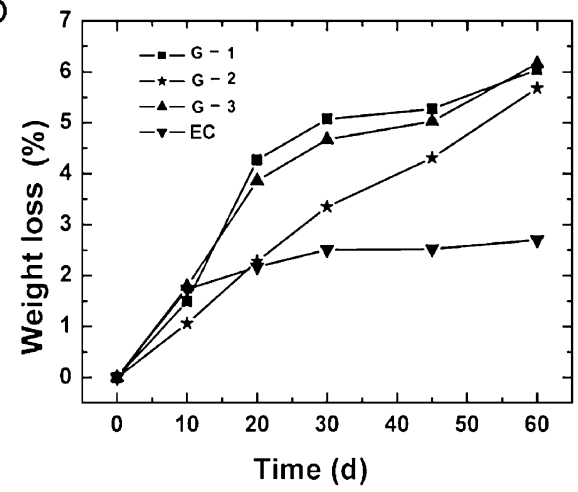

Figure 4. Weight loss of EC and EC-g-PCLA with time in soil (a) and in sludge (b).

PCL homopolymers but the elongation of the former is much smaller that latter.

\section{Biodegradable Properties}

Since EC is a cellulose ether, the biodegradable properties of its copolymer EC-g-PCLA are very attractive aspects for the potential applications. Figure 4 shows the weight loss of the grafting copolymers during the biodegradation in outdoor soil burial (Figure 4a) and in active sludge (Figure 4b) at the temperature of $25-35^{\circ} \mathrm{C}$. From Figure 4 , the weight loss of EC is $2.54 \%$ and $2.70 \%$ after $60 \mathrm{~d}$ in the natural soil condition and in active sludge, respectively. However, the corresponding weight loss of EC- $g$-PCLA copolymer (G-3) increases to $5.09 \%$ and $6.16 \%$, respectively. It is obvious that both EC and the graft copolymer exhibit biodegradability in the natural soil condition and in active sludge to a certain extent. The biodegradability of the copolymer is better than that of EC. Meanwhile, the biodegradability of both EC and the copolymer in active sludge is superior to that in the natural soil condition. It might be expected that there are more microorganisms in the active sludge, which have active effects on the biodegradation of EC and the graft copolymer.

Figure 5 gives the variations of the intrinsic viscosity of EC- $g$-PCLA copolymers with the variation of the grafting percentage before and after biodegradation in outdoor soil (Figure 5a) and active sludge (Figure 5b). It can be found that the intrinsic viscosity of EC- $g$-PCLA is increased with biodegradation and ascends to the maximum after $10 \mathrm{~d}$, 
a

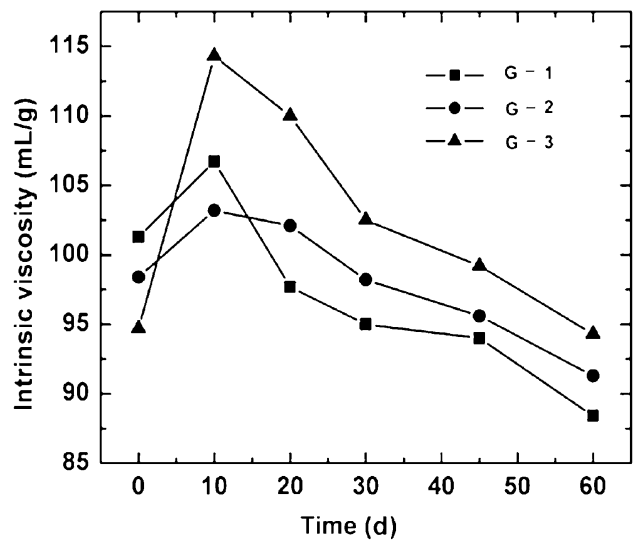

b

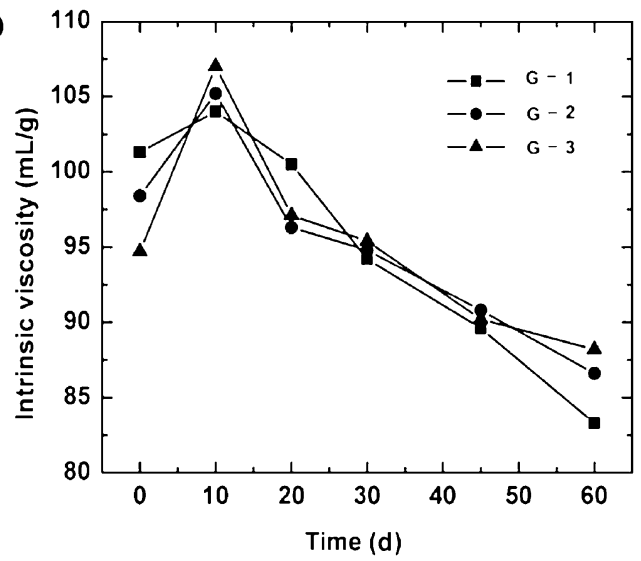

Figure 5. Intrinsic viscosity of graft copolymers before and after biodegradation in soil (a) and in sludge (b).

and then it is decreased with further biodegradation in both conditions. It is implied that the low molecular mass components of EC- $g$-PCLA copolymers are degraded preferentially in the initial stages, which results in the increase of intrinsic viscosity of the remnant copolymers. Then high molecular parts of EC- $g$-PCLA are degraded with further proceeding of degradation, which results in decrease of the intrinsic viscosity of the remnant polymers.

\section{CONCLUSIONS}

Ethylcellulose grafting polycaprolactone monoacrylate copolymers (EC- $g$-PCLA) were synthesized by two-step method. The structure of the graft copolymers was confirmed by FT-IR,
GPC, ${ }^{1} \mathrm{H}$ NMR and ${ }^{13} \mathrm{C}$ NMR. The tensile strength of the EC$g$-PCLA copolymers is decreased but the elongation is increased with increasing the graft percentage $\mathrm{G}$. The flow temperature of the graft copolymers is lower than that of the pure EC. Outdoor soil and sludge tests indicate that the graft copolymers have biodegradability in natural conditions to a certain extent.

Acknowledgment. The financial supports by the National Natural Science Foundation of China (Grant Nos. 50473057 and 50521302) are appreciated.

Received: February 1, 2008

Accepted: October 2, 2008

Published: November 19, 2008

\section{REFERENCES}

1. L. N. Zhang, G. Yang, and H. Q. Liu, Cellulose Commun., 6, 92 (1999).

2. J. Simon, H. P. Muller, and V. Muller, Polym. Degrad. Stab., 59, 110 (1998).

3. L. M. Zhang, Y. B. Tan, and Z. M. Li, Polym. Int., 48, 921 (1999).

4. I. C. Eromosele and T. J. Hamgadu, J. Appl. Polym. Sci., 50, 645 (1993).

5. V. Stepan, H. Krizova, and I. Kossler, Cellul. Chem. Technol., 16 159 (1982).

6. D. S. Wang, Y. Huang, and J. R. Shen, J. Appl. Polym. Sci., 89, 85 (2003).

7. J. N. Chen, Y. F. Huang, and S. F. Liu, J. Appl. Polym. Sci., 42, 3262 (1991).

8. E. A. Abdel-Razik, J. Photochem. Photobiol. Part A: Chem., 107, 271 (1997).

9. D. W. Shen, H. Yu, and Y. Huang, J. Polym. Sci., Part A: Polym. Chem., 43, 4099 (2005).

10. Y. A. Aggour and E. A. Abdel-Razik, Eur. Polym. J., 35, 2225 (1999).

11. Y. A. Aggour, J. Polym. Mater., 16, 1 (1999).

12. Y. Q. Zhang, A. M. Chen, and Y. Huang, Cellul. Chem. Technol., 34, $51(2000)$.

13. W. W. Y. Lau and J. Q. Pan, Polym. Degrad. Stab., 60, 460 (1998).

14. J. Q. Pan, N. C. Liu, and W. W. Y. Lau, Polym. Degrad. Stab., 62, 166 (1998).

15. N. C. Liu, J. Q. Pan, and W. W. Y. Lau, Polym. Degrad. Stab., 62, 308 (1998)

16. D. Gagnaire, D. Mancier, and M. J. Vincendon, J. Polym. Sci., Polym. Chem. Ed., 18, 15 (1980).

17. E. J. Choi, C. H. Kim, and J. K. Park, Macromolecules, 32, 7404 (1999).

18. T. Miyamoto and H. J. Inagaki, J. Polym. Sci., Polym. Chem. Ed., 22, 2365 (1984).

19. W. Z. Yuan, J. Y. Yuan, F. B. Zhang, and X. M. Xie, Biomacromolecules, 8, 1105 (2007). 